\title{
De novo transcriptome sequencing and digital gene expression analysis predict biosynthetic pathway of rhynchophylline and isorhynchophylline from Uncaria rhynchophylla, a non-model plant with potent anti-alzheimer's properties
}

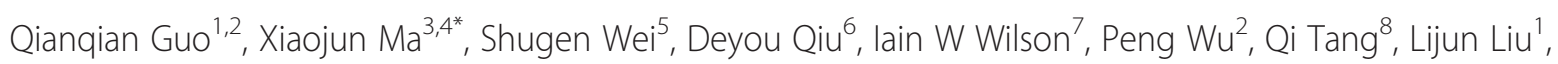
Shoukun Dong ${ }^{1}$ and Wei $\mathrm{Zu}^{1 *}$

\begin{abstract}
Background: The major medicinal alkaloids isolated from Uncaria rhynchophylla (gouteng in chinese) capsules are rhynchophylline (RIN) and isorhynchophylline (IRN). Extracts containing these terpene indole alkaloids (TIAs) can inhibit the formation and destabilize preformed fibrils of amyloid $\beta$ protein (a pathological marker of Alzheimer's disease), and have been shown to improve the cognitive function of mice with Alzheimer-like symptoms.

The biosynthetic pathways of RIN and IRN are largely unknown.

Results: In this study, RNA-sequencing of pooled Uncaria capsules RNA samples taken at three developmental stages that accumulate different amount of RIN and IRN was performed. More than 50 million high-quality reads from a cDNA library were generated and de novo assembled. Sequences for all of the known enzymes involved in TIAs synthesis were identified. Additionally, 193 cytochrome P450 (CYP450), 280 methyltransferase and 144 isomerase genes were identified, that are potential candidates for enzymes involved in RIN and IRN synthesis. Digital gene expression profile (DGE) analysis was performed on the three capsule developmental stages, and based on genes possessing expression profiles consistent with RIN and IRN levels; four CYP450s, three methyltransferases and three isomerases were identified as the candidates most likely to be involved in the later steps of RIN and IRN biosynthesis.

Conclusion: A combination of de novo transcriptome assembly and DGE analysis was shown to be a powerful method for identifying genes encoding enzymes potentially involved in the biosynthesis of important secondary metabolites in a non-model plant. The transcriptome data from this study provides an important resource for understanding the formation of major bioactive constituents in the capsule extract from Uncaria, and provides information that may aid in metabolic engineering to increase yields of these important alkaloids.
\end{abstract}

Keywords: Transcriptome, DGE, Uncaria rhynchophylla, Rhynchophylline, Isorhynchophylline, Terpene indole alkaloid biosynthesis

\footnotetext{
*Correspondence: xjma@public.bta.net.cn; zuweilucky@163.com

${ }^{3}$ Institute of Medicinal Plant Development, Chinese Academy of Medical

Sciences, Beijing 100193, China

'College of Agriculture, Northeast Agricultural University, Harbin 150030,

China

Full list of author information is available at the end of the article
} 


\section{Background}

Alzheimer is a common disease that threatens the health of the elderly [1-3]. Uncaria a member of the Rubiaceae family, has long been used in traditional Chinese medicine to treat hypertension, convulsions, tremor, and stroke [4]. Indole alkaloids present in aqueous solution have been shown to interfere with fiber formation of $\beta$-amyloid protein $(A \beta)$ and destabilize preformed $A \beta$ fibrils, a pathological marker of Alzheimer's disease [5]. An ethanol extract of Uncaria was found to improve the cognitive impairment of Alzheimer's disease caused by D-galactose in mice [6]. RIN and IRN two major alkaloid chemicals that are synthesized in Uncaria capsules, can inhibit the activation of microglia and reduce $A \beta$-induced death in cell lines used to study neuronal differentiation (PC12) [7]. Also RIN reduces $A \beta$ cytotoxicity by inhibiting intracellular calcium overloading and tau protein hyperphosphorylation [8,9]. Extracts from Uncaria have the potential for delaying or alleviating the symptoms of Alzheimer's diseases that could significantly improve the quality of life of patients.

RIN and IRN belong to TIAs. TIAs are a diverse class of natural products that comprise over 2000 members, many of which possess significant physiological activity. Most TIAs are found in three dicotyledon plant families: Apocynaceae, Rubiaceae and Loganiaceae, and are thought to be part of a plants chemical defense against pests [10]. TIAs have a wide variety of different molecular structures and biological activities [11]. Notable TIAs include vincristine and vinblastine of Catharanthus roseus that have anti-tumor activity. Vinblastine is clinically used for the treatment of leukemia, Hodgkin's lymphoma and other cancers [12-15]. Ajmaline of Rauwolfia serpentina can block the sodium channel and resist arrhythmia [15-17]. Whereas, Yohimbine is antagonist of $\alpha$-2-adrenoceptor and a potential drug for the treatment of erectile dysfunction $[18,19]$. Camptothecin of Ophiorrhiza pumila and Camptotheca acuminata is a topoisomerase enzyme inhibitor, and has anti-cancer effect [20,21]. Quinine of Cinchona species is a known anti-malarial drug [22,23]. TIAs production in plants is generally low and difficult to synthesize chemically, limiting the utility of these therapeutic chemicals unless production is increased by metabolic engineering [11]. However, metabolic engineering requires knowledge of the TIAs biosynthesis pathway which is only well understood for a few, such as vindoline and ajmaline.

TIAs biosynthesis involve multiple and complex metabolic pathways. All TIAs are derived from tryptophan and secologanin. Tryptophan decarboxylase (TDC; EC 4.1.1.28), a pyridoxal dependent enzyme converts tryptophan to tryptamine. Isopentenyl diphosphate (IPP) the precursor for all terpenoids is produced by the triose phosphate/pyruvate or "non-mevalonate" pathway (MEP/DOXP pathway) [24,25].
In the first committed step of iridoid terpene biosynthesis, geraniol, derived from IPP, is hydroxylated by geraniol-10hydroxylase (G10H; EC 2.5.1.1) [26,27]. Oxidation of the iridotrial aldehyde to the carboxylic acid is followed by esterification and glucosylationto, yield deoxyloganin; subsequent hydroxylation of deoxyloganin yields loganin. Secologanin is then generated by oxidative cleavage of loganin by the enzyme secologanin synthase (SLS; EC 1.3.3.9) [28-33]. Biosynthesis of secologanin is thought to be potential rate-limiting step in TIAs biosynthesis. Tryptamine of tryptophan metabolic pathway via strictosidine synthase (STR; EC 4.3.3.2) catalysis forms strictosidine [34], and then enters TIAs biosynthetic pathway [35]. This part of the synthesis processes is the best characterized, and the genes encoding the enzymes are known. However each individual TIAs has a synthetic branch, which is started at strictosidine. Through four variable precursors, (4,21-Dehy drogeissoschizine, cathenamine, 19-epi-cathenamine and stemmadenine), each active substance enters the branch path. From structural analysis of RIN and IRN, they may be transformed from Stemmadenine (Figure 1). This speculation is according to the up-stream pathway of TIAs synthesis, which is the biosynthetic process of secologanin [36,37]. Stemmadenine synthesized RIN and IRN through the chemical reactions of oxidation and methyl transfer. This process requires three kinds of enzymes; oxidoreductases, methyltransferases and isomerases. The genes coding for oxidoreductases, methyltransferases and isomerases are defined as "late step genes" of RIN and IRN biosynthesis.

Next generation sequencing technologies have proved to be rapid and cost-effective means to analyze the genome and transcriptome in non-model species. In this study, knowledge of the rapid accumulation periods of RIN and IRN in Uncaria was used to find potential candidates in the synthesis of these TIAs. Using transcriptome sequencing, de novo assembly and DGE analysis, candidate genes encoding putative enzymes responsible for late steps in RIN and IRN synthesis were identified. Our results lay a foundation for construction of the RIN and IRN biosynthetic pathways that will in turn aid the study of the regulation and metabolic engineering of RIN and IRN biosynthesis.

\section{Results}

\section{De novo transcriptome sequencing of capsule}

We compared RIN and IRN content throughout the development of Uncaria's capsule, from fallen petal (designated capsule 1 , sample point \#1) to maturity (designated capsule 3, sample point \#5) (Figure 2). RIN and IRN content changed dramatically during the development of the Uncaria capsule. The two TIAs accumulate in the capsules were detected in capsule 1 (fallen petal), until a maximum is reached at sample point 3 (designated capsule 2) 20 days later, and then drops to its lowest levels at capsule 


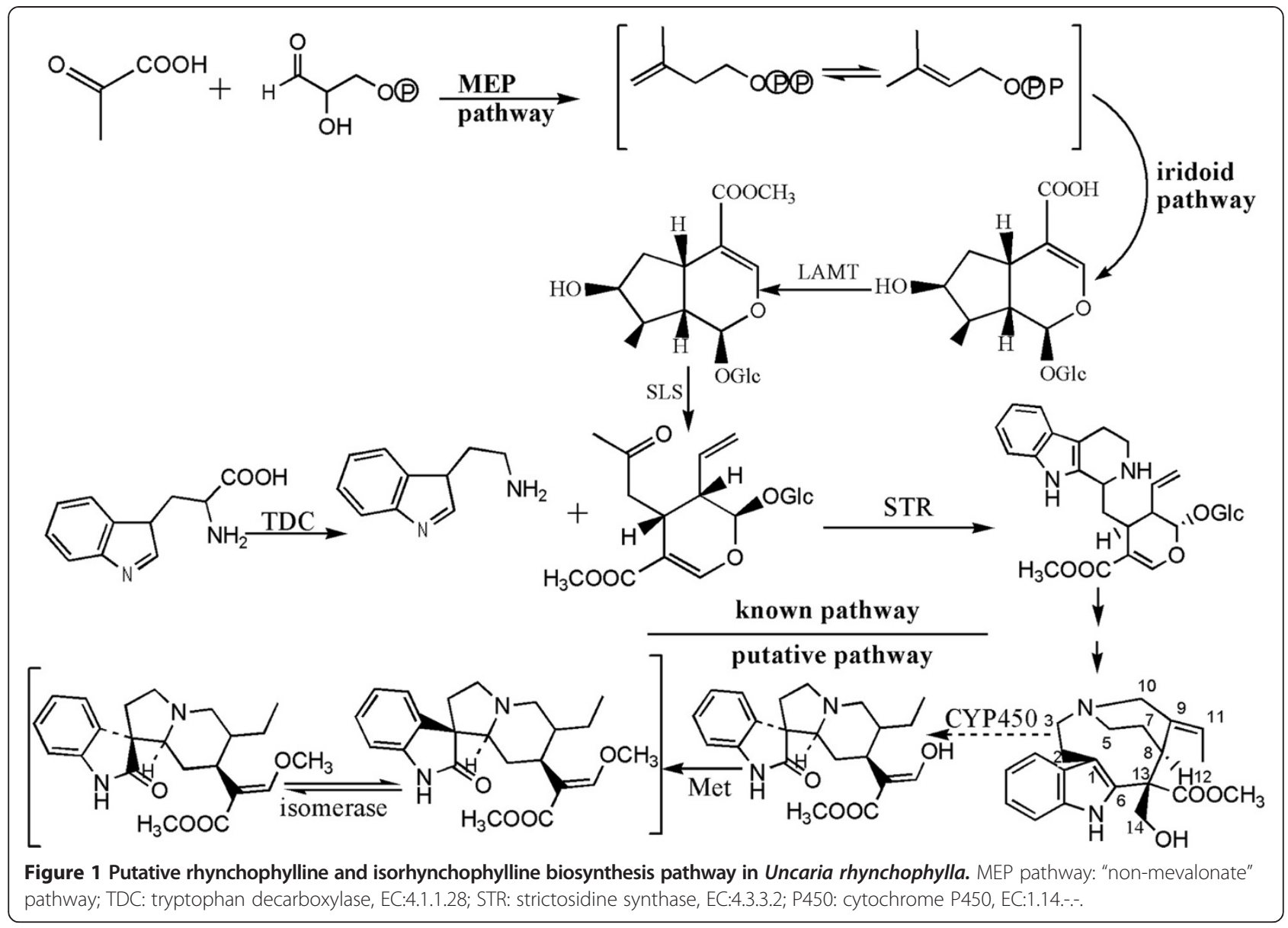

maturity (designated capsule 3) after another 20 days (Figure 2). To obtain an overview of the Uncaria capsule transcriptome in the process of RIN and IRN accumulation, a cDNA library was generated from an equal mixture of RNA isolated from capsule 1 (starting point for RIN and IRN accumulation), capsule 2 (peak RIN and IRN content), and capsule 3 (RIN and IRN content is at its lowest). The library was sequenced using the Illumina HiSeq ${ }^{\mathrm{Tm}}$ 2000 platform. 51 million raw reads were obtained which had 50,159,521 clean reads ( $97.49 \%$ of all raw reads) (in Additional file 1: Table S1), which were used for all subsequent analysis.

With the increase of the sequencing length, sequencing error rates will rise [38,39], but the error rate does not exceed 1\% (in Additional file 2: Figure S1). 6 bp random primers were used in reverse transcription. The incomplete binding of random primers and template could cause sequencing errors of the first six nucleotides position $[39,40]$ (in Additional file 2: Figure S1, S2). Clean reads were spliced into transcripts by trinity software (v2012-10-05) [41], and 100,940 transcripts were obtained. Transcript length was an index which measured with splicing effect. To a certain extent, N50 can evaluate splicing integrity [42]. The average length of transcript was $1,181 \mathrm{bp}$, the longest transcript was $14,101 \mathrm{bp}$, the shortest transcript was 201 bp (N50 was 1970 bp, N90 was 503 bp). From these transcripts, 55,523 unigenes were identified, with an average length of $717 \mathrm{bp}$, the longest unigene was 13,307 bp, the shortest unigene $201 \mathrm{bp}$ (N50 was $1242 \mathrm{bp}$, N90 was $280 \mathrm{bp}$ ). The length statistics of spliced transcripts and unigenes were shown in Additional file 1: Table S2.

\section{Annotation of gene function and CDS prediction}

In order to assign accurate annotation information to unigenes, multiple databases were interrogated including; the NCBI database Non-redundant protein sequences $(\mathrm{Nr})$, the manually annotated and curated protein sequence database (Swiss Prot), NCBI nucleotide sequences (Nt), Protein Family (Pfam), Clusters of Orthologous Groups of proteins (COG), Gene Ontology (GO) and Kyoto Encyclopedia of Genes and Genomes Ortholog database (KO). Annotation results of unigenes are shown in Table 1.

GO is a classification system to describe the properties of the organism genes and their products, including Biological Process, Cellular Component and Molecular Function [43]. Through alignment of GO databases, 26,692 unigenes were annotated to 57 terms of GO classification (Figure 3). Among Biological Process, 'immune 

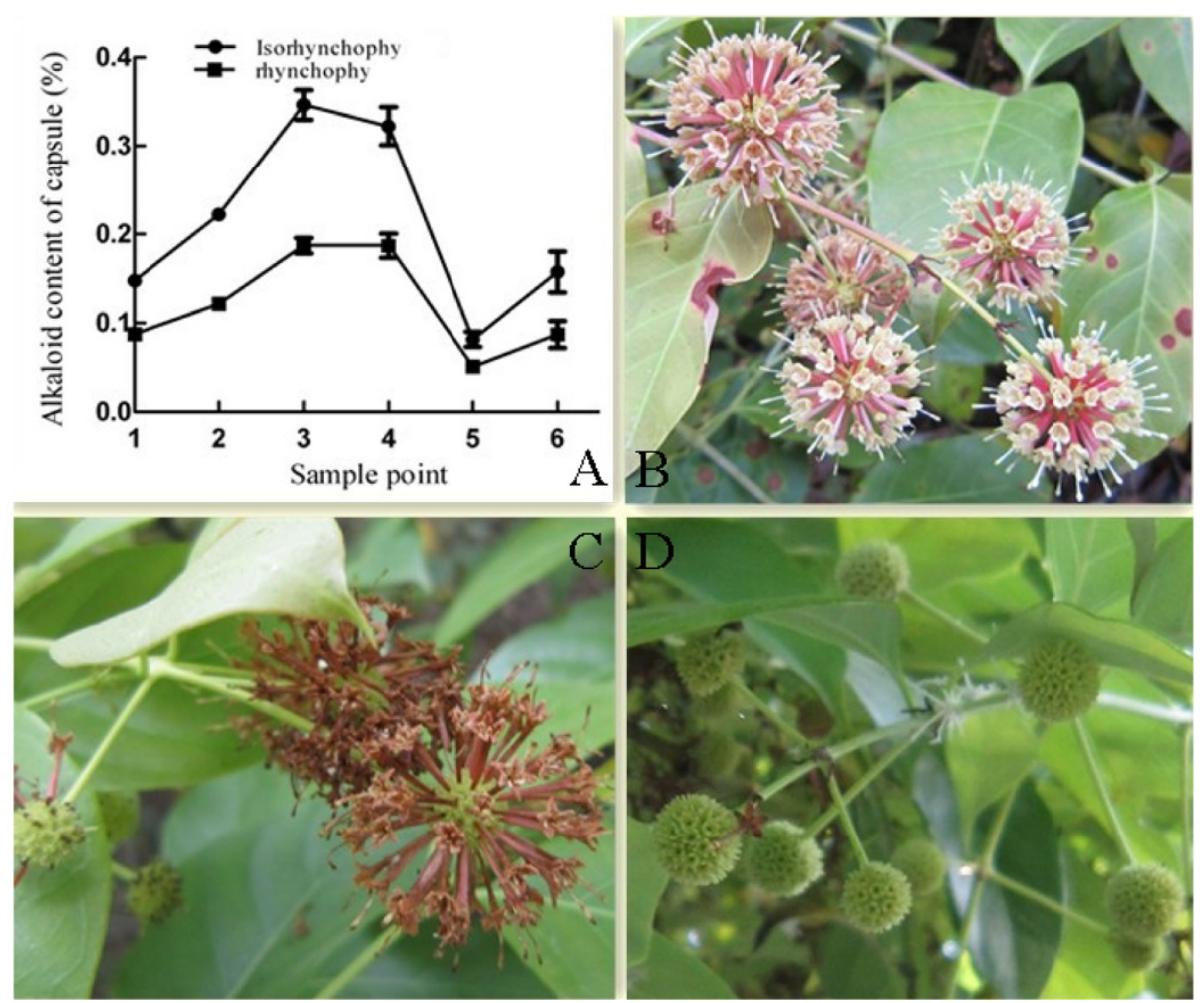

Figure 2 The RIN and IRN content changes of Uncaria capsule from the fallen petal to yellow maturity (A) and biomorph of the sample point $1,3,5(B, C, D)$.

system process [GO:0002376]' was annotated with 637 unigenes, 'response to stimulus [GO:0050896]' was annotated with 6328 unigenes, 'metabolic process [GO:0008152]' was annotated with 15636 unigenes.

KOG is a classification system based on orthologous genes. Orthologous genes have the same function and common ancestor. 11,150 unigenes were annotated to 26 groups by KOG database (Figure 4). (R) General Functional Prediction Only annotated 1869 unigenes at most, and $(\mathrm{X})$ Unnamed protein annotated 2 unigenes at least. (G) Carbohydrate metabolism and transport was annotated with 607 unigenes, (V) Defense mechanisms was annotated with 113 unigenes (Figure 4).

Table 1 Annotation result statistics between unigenes and databases

\begin{tabular}{ccc}
\hline Databases & Number of Unigene & Percent (\%) \\
\hline $\mathrm{Nr}$ & 28,715 & 51.72 \\
Swiss-Prot & 19,984 & 35.99 \\
$\mathrm{Nt}$ & 10,863 & 19.56 \\
$\mathrm{Pfam}$ & 21,083 & 37.97 \\
$\mathrm{COG}$ & 11,150 & 20.08 \\
$\mathrm{GO}$ & 26,692 & 48.07 \\
$\mathrm{KO}$ & 12,256 & 22.07 \\
\hline
\end{tabular}

$\mathrm{KO}$ is a network diagram of cell metabolic pathways, including metabolism, genetic information processing, environmental information processing, cellular processes, organismal systems and human diseases. In this study, 12,256 unigenes were annotated by alignment with the $\mathrm{KO}$ database. Among them; 4,326 unigenes were annotated to Metabolism (35.30\% of the total), 660 to Environmental Information Processing (5.39\%), 2,060 to Genetic Information Processing (16.80\%), 1,340 to Organismal Systems (10.93\%), 955 to Cellular Processes (7.79\%). According to the $\mathrm{KO}$ annotation, removing unigenes associated with the Human Diseases, the remaining 8,745 unigenes (15.75\% of all unigenes) were classified to 31 KEGG pathways (Figure 5). Among them; 269 were annotated to Metabolism of terpenoids and polyketides pathway, 74 unigenes involved in Terpenoid backbone biosynthesis [PATHWAY: ko00900], 17 unigenes involved in Monoterpenoid biosynthesis [PATHWAY: ko00902], 593 unigenes were annotated to Amino acid metabolism pathway, 54 unigenes involved in Phenylalanine, tyrosine and tryptophan biosynthesis [PATHWAY: ko00400], 295 unigenes were annotated to Biosynthesis of other secondary metabolites pathway, 8 unigenes involved in Indole alkaloid biosynthesis [PATHWAY: ko00901].

Open Reading Frame (ORF) was obtained by comparing annotation results of unigenes with the different 


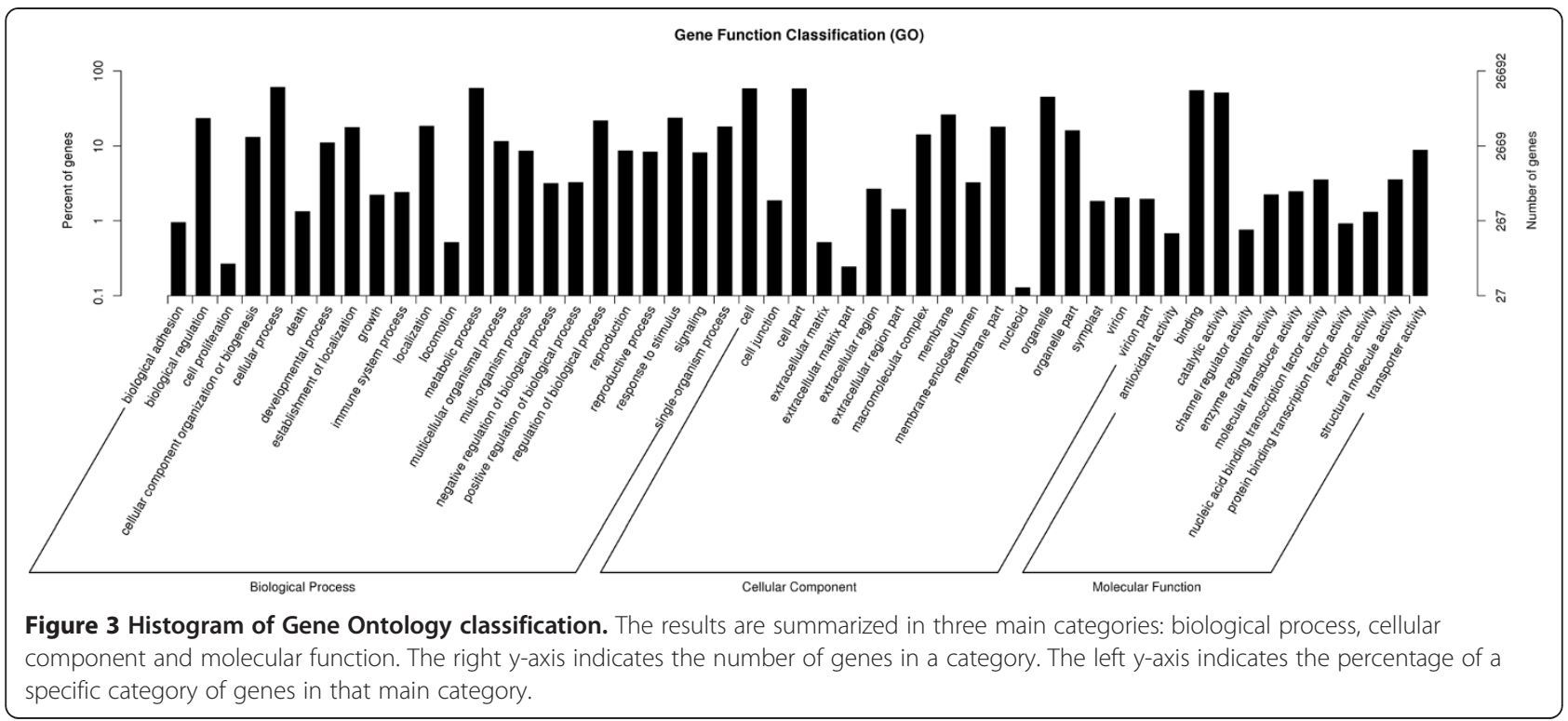

databases, the unigenes were then determined using Coding Sequence (CDS) according to the standard codon table [38]. If the results from different databases were contradictory, priority was given to Nr, Swiss-prot and KEGG GENES respectively. The unigenes that failed to match the database information the predicted protein CDS was determined using estscan software [44]. The CDS of 28,611 unigenes (51.52\% of all unigenes) could be predicted using the annotation databases whereas 16,566 unigenes $(29.84 \%$ of all unigenes) CDS predictions were made using estscan software.

\section{Genes involved in the biosynthesis of TIAs}

TIAs biosynthesis involves three upstream metabolic pathways, including MEP pathway, iridoid glycosides pathway and shikimate pathway [45]. Secologanin is a natural active substance and iridoid glycoside product of TIAs biosynthetic precursor, but its synthesis process is not yet understood. Another precursor tryptamine; is generated by tryptophan using the shikimate pathway by catalysis with TDC. TDC is communication node between primary metabolism and secondary metabolism. Unigenes annotated by comparison with the $\mathrm{Nr}$ database comparison that could encode enzymes related the above pathways are listed in Additional file 1: Table S3. All putative oxidoreductases that could represent genes involved in the late steps in RIN and IRN synthesis are listed in Additional file 1: Table S4 (A-C).

The MEP pathway is branch of Terpenoid backbone biosynthesis [PATHWAY: ko00900] and the iridoid glycosides pathway is branch of Monoterpenoid biosynthesis [PATHWAY: ko00902], The product of phenylalanine, tyrosine and tryptophan biosynthesis [PATHWAY: ko00400] enters indole alkaloid biosynthesis [PATHWAY: ko00901] via
TDC and STR. Putative unigenes associated with TIAs synthesis were annotated by $\mathrm{KO}$ database (in Additional file 1: Table S5). The Uncaria capsule transcriptome data contained all genes encoding every enzyme of the MEP pathway and shikimate pathway. However, there are deficiencies associated with the databases as the Monoterpenoid biosynthesis [PATHWAY: ko00902] in $\mathrm{KO}$ is only aligned to myrcene/ocimene synthase (EC 4.2.3.15). In the KEGG pathway, TDC belongs to indole alkaloid biosynthesis [PATHWAY: ko00901], which is only aligned to STR, not aligned to the TDC. The reason may be few studies were conducted with TIAs biosynthesis, and reference data was insufficient.

\section{Capsule DGE analysis at different periods}

DGE sequencing analysis of capsule 1, capsule 2 and capsule 3 was performed by Illumina HiSeq 2000 sequencing platforms, respectively. Capsule 1 produced 9,270,290 clean reads, capsule 2 produced 13,298,879 clean reads, and capsule 3 produced 15,331,847 clean reads. The clean reads of the samples were mapped to the reference sequence derived from our Uncaria transcriptome data, and obtained from the annotation information of each sample using RSEM software [46]. Total mapped reads were capsule $18,529,453$ (92\%), capsule 2 12,258,147 (92\%), and capsule $314,047,840$ (92\%) with approximately 47,000 unigenes annotated in each sample. To estimate the level of gene expression the reads count were transformed into Reads Per Kilo bases per Million mapped Reads (RPKM) [47]. RPKM is the number of reads per million reads from one gene per thousand bases, considering the effect of sequence length and depth of RNA-seq process on the reads count. The size of RPKM value therefore reflects the abundance of 


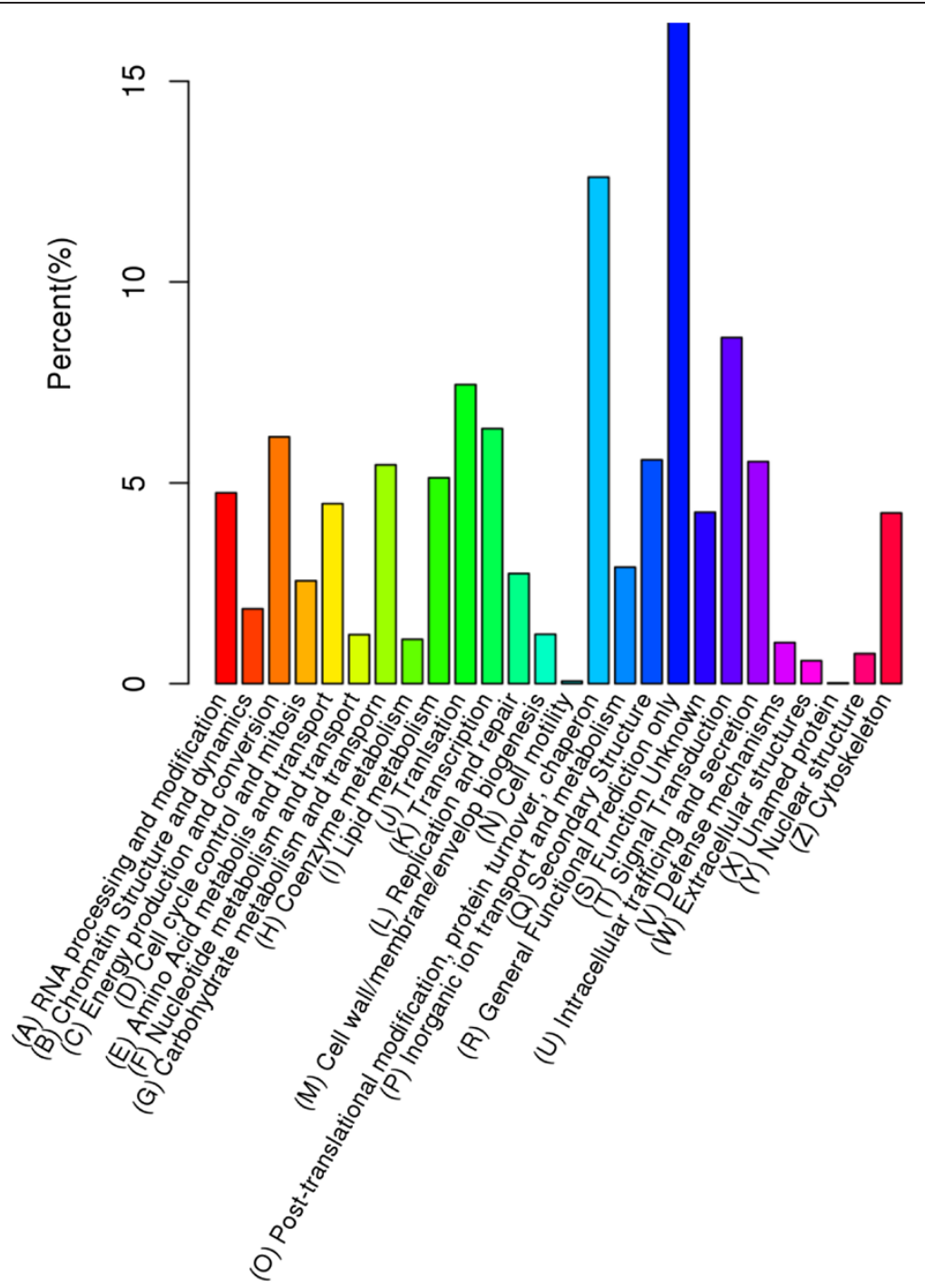

Figure 4 Histogram of unigene KOG classification. The $x$-axis indicates 26 groups of KOG. The $y$-axis indicates the percentage of the number of genes annotation under the group in the total number of genes annotation.

the gene expression and we defined RPKM $>0.3$ as the threshold of significant gene expression. The density distribution of RPKM can test gene expression profiles of samples; comparison chart of gene expression profiles among three samples was showed in Figure 6. Among them, the common genes of three samples reached 38,592 , characteristic genes of capsule 1 were 1,687 , characteristic genes of capsule 2 were 1,901 and characteristic genes of capsule 3 were 1,986.

\section{Analysis of differentially expressed genes}

Read count data was used to analyze the difference of gene expression. Since only one biological samples was sequenced, in order to avoid possible high false positive rates we first used trimmed mean of $M$ values (TMM) to standardize data processing [48], then took advantage of DEGseq to analyze differentially expressed genes; the analysis conditions were qvalue $<0.005 \& \log 2$ (foldchange) $>1$ [49-51]. There were 1,488 differentially expressed genes identified between capsule 2 and capsule 1, with 803 genes were up-regulated and 685 genes were down-regulated; 1,343 differentially expressed genes between capsule 3 and capsule 1, 749 genes were up-regulated and 549 genes were down-regulated; 1,779 differentially expressed genes in capsule 3 vs capsule 2 , with 865 genes were up-regulated and 914 genes were downregulated. Comparison between differentially expressed genes between the three samples is shown in Figure 7. Capsule 3 vs capsule 2 comparisons had the most specific differentially expressed genes. Interestingly, capsule 3 vs capsule 2 and capsule 2 vs capsule 1 had 748 common differentially expressed genes, capsule 3 vs capsule 


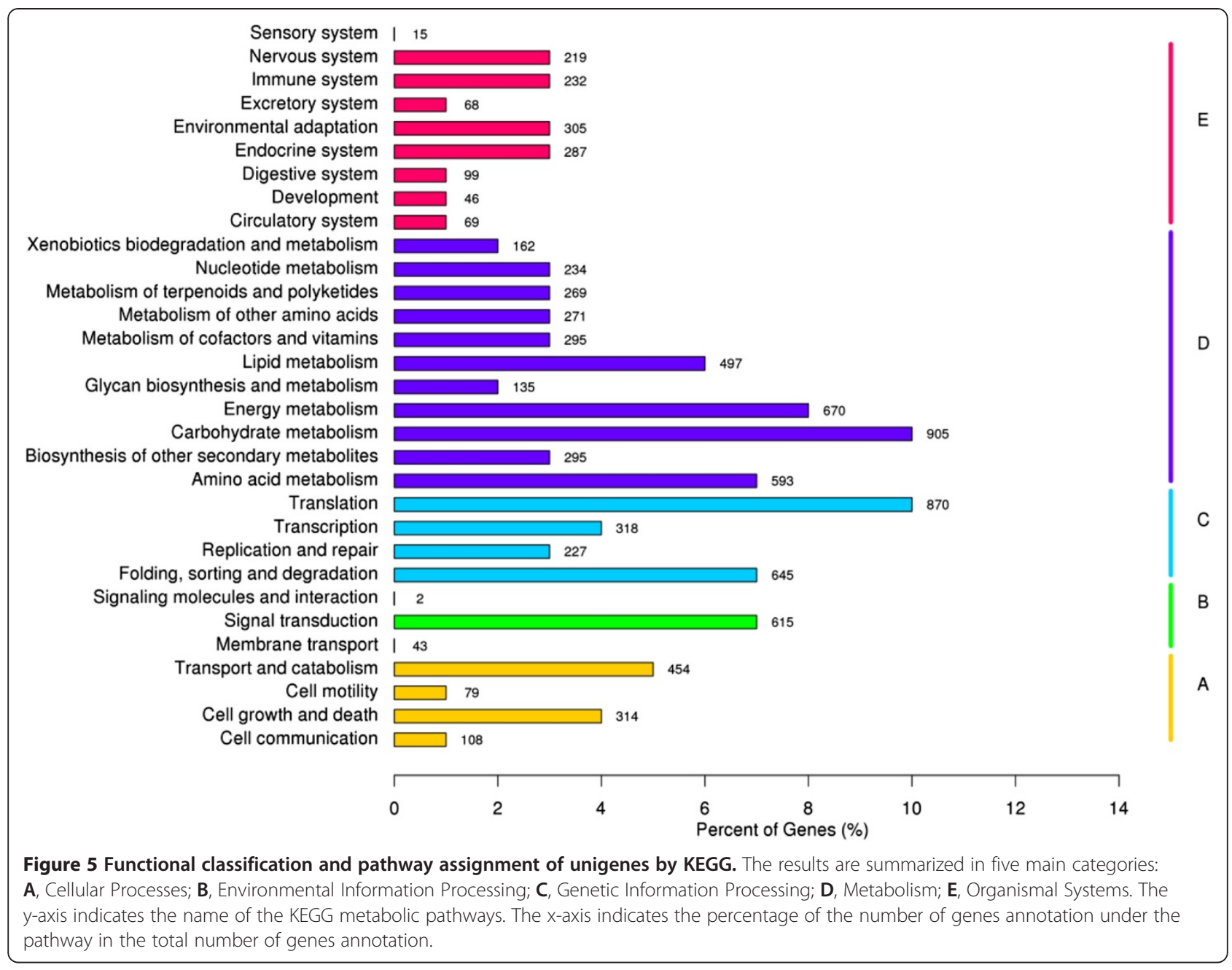

2 and capsule 3 vs capsule 1 had 744 common differentially expressed genes, the two were very close.

Differentially expressed genes of from the DGE analysis were further analyzed using GO and KEGG enrichment in order to determine their potential function and within a metabolic pathway. GO enrichment classification using GOseq [52]. GO classification of differentially expressed genes between the different capsule stages is showed in Additional file 2: Figure S3 (A-C). 419 differentially expressed genes of capsule 2 vs capsule 1 were classified into the biological process, and 133 genes were identified as involved in oxidation-reduction process [GO: 0055114]. 703 genes were classified into cellular component. 903 genes were classified into molecular function, 903 genes involved in catalytic activity [GO:0003824], 137 genes involved in oxidoreductase activity [GO: 0016491]. 588 genes among differentially expressed genes of capsule 3 vs capsule 1 were classified into the biological process, 226 genes involved in oxidation-reduction process [GO: 0055114]. 507 genes were classified into cellular component, 765 genes were classified into molecular function, 240 genes participated in oxidoreductase activity [GO: 0016491]. 657 genes among differentially expressed genes of capsule 3 vs capsule 2 were classified into the biological process, 255 genes involved in oxidation-reduction process [GO: 0055114]. 1301 genes were classified into cellular component, 841 genes were classified into molecular function, 272 genes participated in oxidoreductase activity [GO: 0016491].

KEGG enrichment classification (hypergeometric test) was used to identify significant enrichment pathways of differentially expressed genes between the different stages of Uncaria capsule development using a False Discovery Rate (FDR) $\leq 0.05$. Flavonoid biosynthesis [PATHWAY: ko00941], phenylpropanoid biosynthesis [PATHWAY: ko00940], phenylalanine metabolism [PATHWAY: ko00360] and biosynthesis of secondary metabolites [PATHWAY: ko01110] were significantly enriched pathways among the differentially expressed genes between the capsule stages. Indole alkaloid biosynthesis [PATHWAY:ko00901] was enriched in capsule 3 vs capsule 2, but did not reach significant level (FDR $\leq 0.05)$ [in Additional file 2: Figure S4 (A-C)]. 


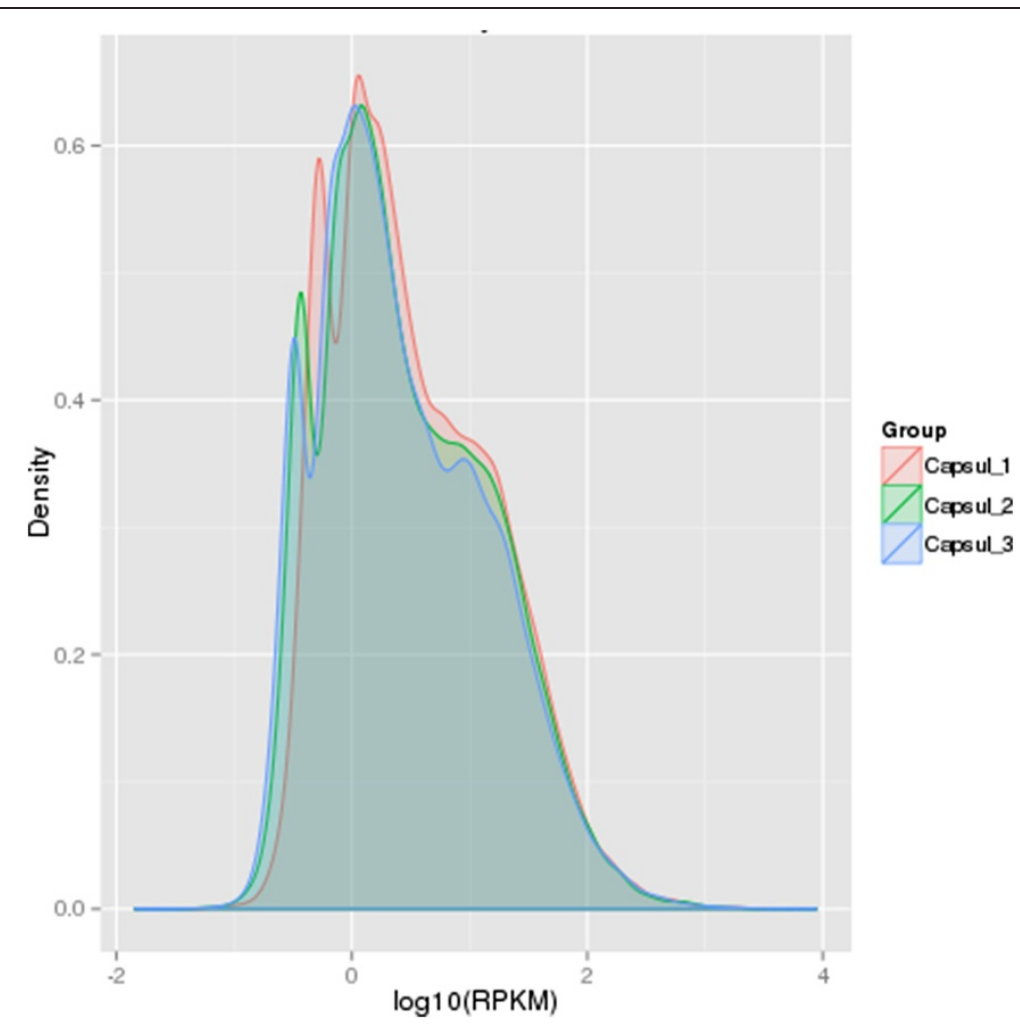

Figure 6 Capsule RPKM density distribution at different periods. The $y$-axis indicates the density values of $\log _{10}$ (RPKM). The $x$-axis indicates the $\log _{10}($ RPKM) values of genes. Red, green and blue represent Capsule 1, Capsule 2 and Capsule 3 respectively.

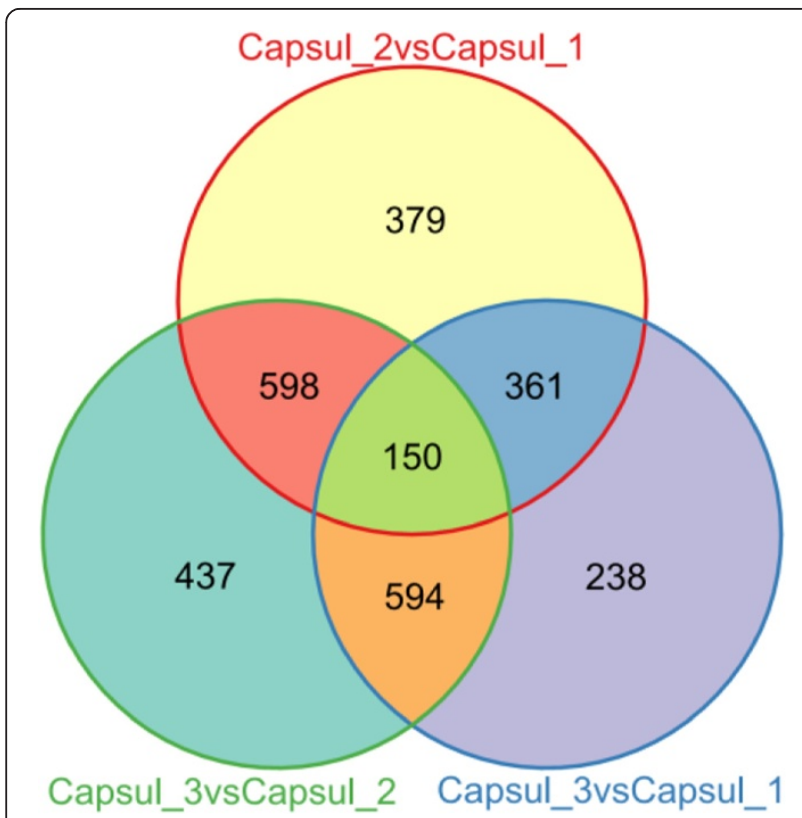

Figure 7 Venn diagram of differentially expressed genes. The sum of the numbers in each large circle represents total number of differentially expressed genes between combinations, the overlap part of the circles represents common differentially expressed genes between combinations.

\section{Analysis of genes with homology to known genes involved in TIA biosynthesis}

There were 68 unigenes that may be involved in the TIA biosynthesis due to their homology to genes known to be involved in TIA from other species by NCBI Nr comparisons [53]. To narrow down this list we looked for genes that showed expression profiles consistent with changes in RIN and IRN content during capsule development. As previously mentioned RIN and IRN content is strongly elevated between capsule 1 and capsule 2, then decreased significantly between capsule 2 and capsule 3. RIN and IRN content is also lower in capsule 3 than that present in capsule 1.17 of the putative TIAs biosynthesis genes showed significantly up or downregulated expression (in Additional file 1: Table S6). However, only ten genes had expression profiles either similar or inverse to RIN and IRN accumulation in at least 2 stages. Anthranilate synthase (AS; EC 4.1.3.27) (comp12708_c0) and loganin acid methyltransferase (LAMT; EC 2.1.1.50) (comp33316_c0) expression profiles matched of the direction of RIN and IRN content change, whereas SLS (comp35216_c0) had the inverse expression profiles. The expression profiles of TDC (comp34949_c0), G10H (comp33830_c0) and STR (comp16908_c0 and comp38465_c1) had two phases 
consistent with RIN and IRN changes. A total of 64 unigenes of $\mathrm{KO}$ annotation involved in the synthesis of TIAs were based on enzymes of KEGG pathway. DGE analysis found that 11 unigenes showed significantly up/down-regulated expression changes (in Additional file 1: Table S7). AS (comp12708_c0) expression changes with direction of RIN and IRN content change was consistent. The up/down regulated expression changes of 3-dehydroquinate dehydratase/ shikimate dehydrogenase (EC 4.2.1.10) (comp22817_c0) and STR (comp16908_c0) with direction of RIN and IRN content change, two phases were consistent.

\section{Analysis of putative genes involved in the late steps of RIN and IRN biosynthesis}

Enzymes classes putatively involved of biosynthesis of RIN and IRN include oxidoreductase, transferase, and isomerase. CYP450 is one of the oldest protein families, has catalytic oxidation function of carbon-carbon bond, alkyl hydroxylation and hydroxyl oxidation, and plays an important role in plant secondary metabolites synthesis process [54-57]. Oxidoreductases involved in RIN and IRN synthesis are therefore likely to come from the CYP450 family. There are 30 varieties of methyltransferase but only o-methyl transferase is likely to be involved in RIN and IRN [58,59]. Isomerase are relatively unknown, however isomers conversion between RIN and IRN could be realized through enzyme catalysis, as natural transformation of the chemical equilibrium is likely to be small.

From the Uncaria transcriptome data, a total of 193 CYP450, 280 methyltransferase and 144 isomerase unigenes were identified. DGE analysis showed 29 CYP450s were significantly up/down-regulated during capsule development (in Additional file 1: Table S8A). Only the expression of 1 unigene (comp28593_c0) matched changes in RIN and IRN content. The expression profiles of 9 unigenes (comp21921_c0, comp26673_c0, comp30485_c0, comp31075_c0, comp32966_c0, comp34791_c1, comp35950_c0, comp37580_c0 and comp38378_c0) had similar to RIN and IRN content in two stages. 21 methyltransferase unigenes possessed significantly differential expression in the process of capsule development (in Additional file 1: Table S8 B). 1 unigene (comp33316_c0) expression profile was consistent to RIN and IRN content. Whereas, the expression profile of 6 unigenes (comp12388_c0, comp29880_c0, comp34948_c0, comp36199_c0, comp36942_c0, and comp41847_c0) where similar to RIN and IRN content in two stages. 12 unigenes putatively encoding isomerases had significantly altered expression in the process of capsule RIN and IRN content change (in Additional file 1: Table S8C), with 3 unigenes (comp22426_c0, comp28178_c0 and comp38594_c0) showing changes consistent with the TIAs RIN and IRN content in two stages.
STR is a starting point and key enzyme of TIAs synthesis involved in the early part of TIAs synthesis. STR expression will affect the metabolic activities of the downstream branch. The gene expression profiles of TIAs downstream branch are expected to be similar to the expression profiles of STR. The gene expression profiles not only include up/down-regulated expression, but also include gene expression variation and amplitude from each stage of capsule $1 \rightarrow$ capsule $2 \rightarrow$ capsule 3 . Expression profiles of CYP450, methyltransferase and isomerase hierarchically clustered with that of STR $\left(S T R_{\mathrm{Nr}}\right.$ comp16908_c0 and comp38465_c1; STR $\mathrm{KO}$, comp16908_c0 and comp36585_c0) (shown in Figures 8 and 9). Ten candidate genes were found to cluster closely with STR (correlation >0.99). The candidate genes of CYP450 had 4 unigenes (comp31075_c0, comp28593_c0, comp34791_c1 and comp26673_c0); candidate genes of methyltransferase had 3 unigenes (comp31161_c0, comp33316_c0 and comp34948_c0) and candidate genes of isomerase had 3 unigenes (comp22426_c0, comp38594_c0 and comp28178_c0). Through the alignment of transcriptome database, the candidate unigene sequences and amino acid sequences of "late step genes" of RIN and IRN biosynthesis were obtained and showed in Additional file 1: Table S9.

\section{Quantitative PCR analysis of candidate TIA biosynthesis \\ genes}

Quantitative PCR analysis was performed on 13 selected genes from CYP450, methyltransferase, isomerases and STR functional categories putatively involved in TIA biosynthesis of Uncaria. The relative expression changes of the candidate genes are shown in Figure 10. Generally during the developmental process of capsule 1 to capsule 2 , and capsule 2 to capsule 3 growth, the expression profiles of the genes assayed show highest gene expression at the capsule 2 stage, and lowest gene expression at capsule 3, mirroring the measured RIN and IRN content profiles, and confirming the DGE expression data. The STR genes (comp16908_c0, and comp38465_c1) however showed only marginal increases at capsule 2 stage. The relative expression of one of the CYP450 genes (comp26673_c0) was inverse to the expected expression, with lowest expression at capsule 2 and then increasing at capsule 3 (Figure 10 and Additional file 1: Table S8).

\section{Discussion}

The medicinal parts of Uncaria are its "stem and stem hook" and the effective pharmacological constituents are RIN and IRN. In Uncaria plants, if a branch has a capsule, then there will be no stem hook. A branch with a stem hook growing on it, will not have flowers nor capsules next to it. In such case, there are two kinds of leaves and stems, one is the twig containing the capsule 

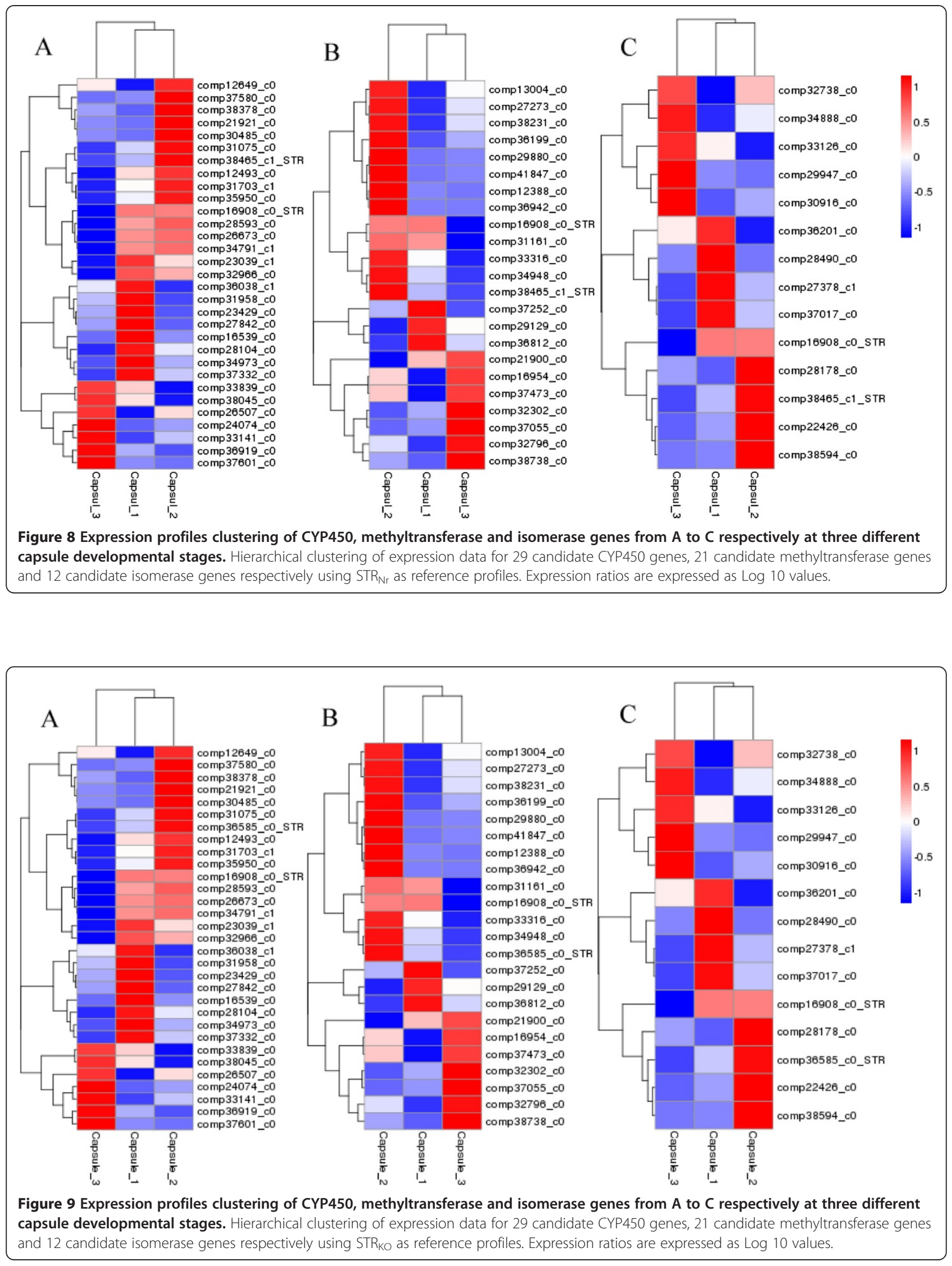

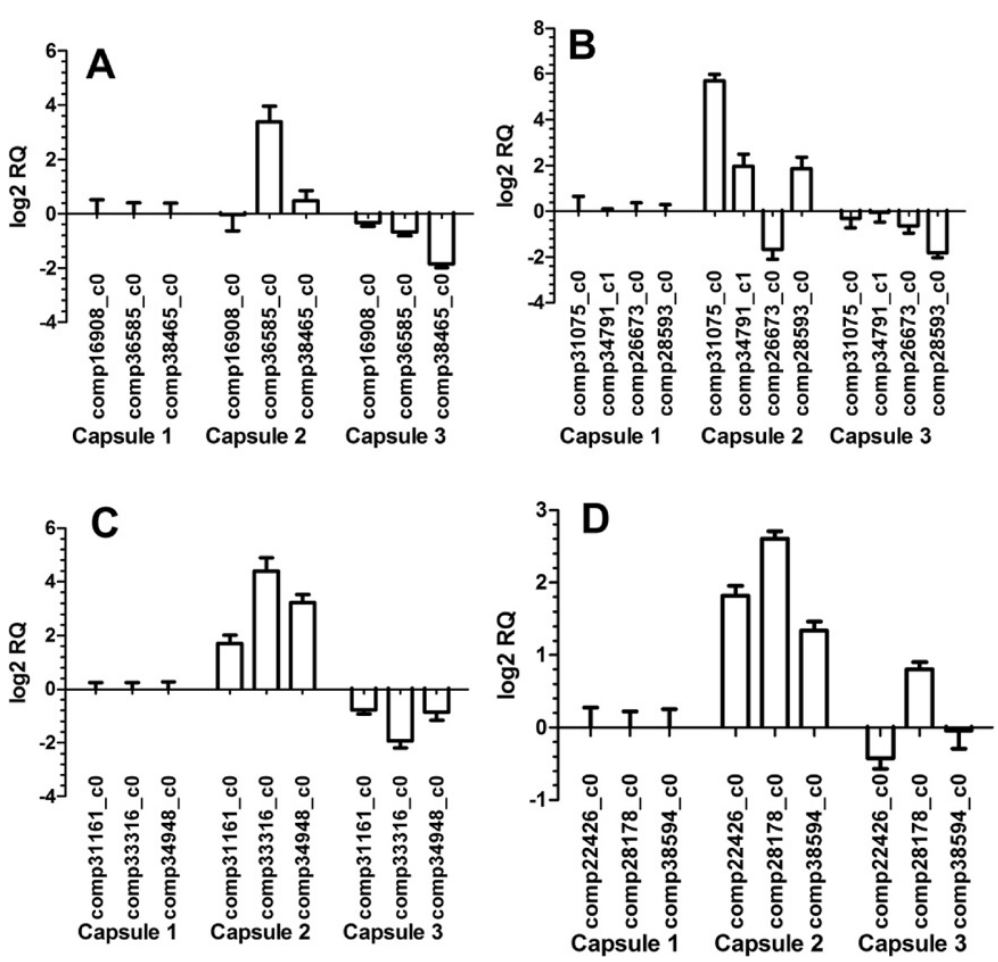

Figure 10 Quantitative PCR analysis of the candidate downstream genes. A STR; B CYP450; C Methyltransferases; D Isomerases.

and the other is the twig containing a stem hook. "Stem and stem hook", leaf, stem and capsule of Uncaria contain RIN and IRN, and different parts contain different content (data not shown). However, the difference between the RIN and IRN content of the "stem and stem hook" and that of stem on the branch with capsule is not significant. In addition, the serious lignification of the stem influences the integrity of RNA. By contrast, the difference between the RIN and IRN content of leaf on the branch with capsule and that of the leaf on the branch with stem hook is significant. No proteins related to the biosynthesis of the two TIAs have been found through dimensional electrophoresis analysis (data not shown). Among all the tissues and organs, the capsule has the highest RIN and IRN content and during the developmental process there is an increase and then decrease in the accumulation of the RIN and IRN. These changes in RIN and IRN content in the capsule reflect different rates of synthesis and provides a means for identifying candidate genes involved in the biosynthesis of RIN and IRN through analyzing the gene expression profiles [60].

Next generation sequencing technology (NGS) offers a shortcut for research on functional genomics, comparative genomics [61-63] and genetic analysis [64] of nonmodel plants. With the progress of NGS, and the increase of sequencing throughput, improvements in accuracy, and decreased costs, enable this technology to be broadly applied to various research areas [65,66]. There is little to no genome information of Uncaria or the related species. Therefore sequencing RNA using NGS from a specific period of plant development or treatment provides the information necessary for de novo assembly of the transcriptome; generating a reference transcriptome sequence for non model plants that can provide the basis for finding genes associated with particular important functions [67-69]. DGE can quickly and thoroughly analyze the gene expression under a variety of tissues and conditions [70]. DGE however requires a reference sequence to align the relative small read lengths. Combining DGE with an RNA-sequencing generated reference transcriptome provides an excellent combination for analysis of non model plant species. Confirmation of the expression profiles of a subset of genes identified DGE using qPCR indicates the accuracy of this method for quickly identifying candidates based on expression profiles.

Predicting the biosynthesis pathway of RIN and IRN lays the theoretical foundation for the regulation of the RIN and IRN accumulation as well as their metabolic engineering research [71]. The formation of TIAs precursor involves the shikimate pathway of tryptophan metabolism and MEP pathway which are the common pathways of terpene biosynthesis. During the comparison and annotation of TIA like genes, we found all the encoding genes of the related enzymes in the two pathways in 
our cDNA library. Moreover, the encoding genes of STR which include the CYP450, methyltransferase and isomerase of later biosynthetic step in RIN and IRN biosynthesis were also found. By regulating the metabolic pathway, the accumulation of the RIN and IRN in the plant can be improved [72,73]. This can be started from many aspects such as improving the TIAs biosynthesis pathway activity and upstream shunt volume of carbon skeleton. Improvement of biosynthesis pathway activity can be fulfilled through improving the activity of the key enzyme in the pathway $[74,75]$. The increase of carbon shunt volume needs to shunt more carbon from the glycometabolism to enter the target pathway. Additionally, weaken or cutting off the carbon channel of non target pathways etc. The MEP pathway exists in the plastid, the amount of which can be increased by employing the matrilineal inheritance feature of plastid. In this way, more carbon in the glycometabolism will enter the RIN and IRN biosynthesis pathway. Alternatively, increase/enhance the transporter quantity/transport ability on the plastid membrane involved in the intermediate products of the MEP pathway of RIN and IRN biosynthesis through transformation of the organelle. Ultimately the substrate amount of iridoid pathway is increased. The auto-regulation mechanism of plant biosynthetic pathways is complex and strict. Construction of the vitro RIN and IRN biosynthesis pathway using information from the related research on vinblastine and vincristine [76-79] may provide a means of accelerating our knowledge of the pathways. The low yield of natural active substances is one of the factors limiting the development and utilization of Chinese medicine resources. The use of engineering techniques to improve production of natural active substance is an effective way to solve the contradiction between supply and demand. This study presented the pathway of RIN and IRN biosynthesis, and laid the theoretical foundation for the medicinal potential development of RIN and IRN. If the yield issues of RIN and IRN are effectively solved then they will have a wider range of applications in the treatment of Alzheimer's disease.

\section{Conclusion}

A combination of De novo transcriptome sequencing and DGE analysis based on the next generation sequencing technology was shown to be a powerful method for identifying candidate genes encoding enzymes responsible for the biosynthesis of novel secondary metabolites in a non-model plant. Through this method, biosynthetic encoding genes of Uncaria TIAs biological precursor were found. CYP450, methyltransferase and isomerase were selected as potential candidates involved in late biosynthesis of RIN and IRN. The transcriptome data from this study provides an important resource for understanding the formation of major bioactive constituents in the capsule extract from Uncaria.

\section{Methods}

\section{Sample collection and preparation}

Uncaria was collected from Nanning, Guangxi, China, and identified as Uncaria rhynchophylla by Ma Xiaojun. From blossom to ripened capsules, the capsules were collected once every ten days and six times totally. The collected capsules were divided into two parts. One part was frozen in liquid nitrogen for isolation of RNA, and stored at $-80^{\circ} \mathrm{C}$ until use. The other part was used for the determination of RIN and IRN content by high performance liquid chromatography after drying and crushing. The plant samples were stored in Plant Physiology Laboratory of Northeast Agricultural University.

The analysis results from high performance liquid chromatography showed a single peak curve of RIN and IRN content changes (Figure 2A) [80]. Sampling point 3 had the highest RIN and IRN content and was designated capsule 2. Sampling point 1 (designated capsule 1) and 5 (designated capsule 3) had the low RIN and IRN content. RNA samples from sample point 1 (capsule 1), 3 (capsule 2) and 5 (capsule 3) were used for highthroughput sequencing.

$2 \mu \mathrm{L}$ from each of three samples were mixed for transcriptome sequencing. $4 \mu \mathrm{L}$ from each of three samples were used for expression profile sequencing. The RNA samples from sample point 1,3 and 5 were named capsule 1 , capsule 2 and capsule 3 in expression profile sequencing. Representative images of sample point 1, 3 and 5 are shown in Figure 2B,C,D.

\section{RNA isolation, quantification and qualification}

Total RNA of capsule 1, capsule 2 and capsule 3 were isolated using the improved CTAB method [81]. RNA degradation and contamination was monitored on $1 \%$ agarose gels. RNA purity was checked using the NanoPhotometer spectrophotometer (IMPLEN, CA, USA). RNA concentration was measured using Qubit ${ }^{\circ}$ RNA Assay Kit in Qubit $^{\circ} 2.0$ Flurometer (Life Technologies, CA, USA). RNA integrity was assessed using the RNA Nano 6000 Assay Kit of the Bioanalyzer 2100 system (Agilent Technologies, CA, USA).

\section{Sample preparation for sequencing}

A total amount of $3 \mu \mathrm{g}$ RNA per sample were used as input material for the RNA sample preparations. All four samples had RNA integrity number values above 8.0. Sequencing libraries were generated using Illumina TruSeq ${ }^{\text {tax }}$ NA Sample Preparation Kit (Illumia, San Diego, USA) following manufacturer's recommendations and four index codes were added to attribute sequences to each sample. Briefly, mRNA was purified from total 
RNA using poly-T oligo-attached magnetic beads. Fragmentation was carried out using divalent cations under elevated temperature in Illumina proprietary fragmentation buffer. First strand cDNA was synthesized using random oligonucleotides and SuperScript II. Second strand cDNA synthesis was subsequently performed using DNA Polymerase I and RNase H. Remaining overhangs were converted into blunt ends via exonuclease/ polymerase activities and enzymes were removed. After adenylation of 3' ends of DNA fragments, Illumina PE adapter oligonucleotides were ligated to prepare for hybridization. In order to select cDNA fragments of preferentially $200 \mathrm{bp}$ in length the library fragments were purified with AMPure XP system (Beckman Coulter, Beverly, USA). DNA fragments with ligated adaptor molecules on both ends were selectively enriched using Illumina PCR Primer Cocktail in a 10 cycle PCR reaction. Products were purified (AMPure XP system) and quantified using the Agilent high sensitivity DNA assay on the Agilent Bioanalyzer 2100 system.

\section{Clustering and sequencing}

The clustering of the index-coded samples was performed on a cBot Cluster Generation System using TruSeq PE Cluster Kit v3-cBot-HS (Illumia) according to the manufacturer's instructions. After cluster generation, the library preparations were sequenced on an Illumina Hiseq 2000 platform.

\section{Quality control}

Raw data (raw reads) of fastq format were firstly processed through our self-written perl scripts. In this step, clean data (clean reads) were obtained by removing reads containing adapter, reads containing ploy- $\mathrm{N}$ and low quality reads from raw data. At the same time, Q20, Q30, GC-content and sequence duplication level of the clean data were calculated. All the downstream analyses were based on clean data with high quality.

\section{Transcriptome assembly}

Pair-end sequencing was performed. The left files (read 1 file) from all libraries/samples were pooled into one big left.fq file, and right files (read 2 file) into one big right.fq file. Transcriptome assembly was accomplished based on the left.fq and right.fq using Trinity [41] with min_kmer_cov set to 2 and all other parameters set default.

\section{Gene functional annotation}

Gene function was annotated based on the following databases: $\mathrm{Nr}$ (NCBI non-redundant protein sequences); $\mathrm{Nt}$ (NCBI non-redundant nucleotide sequences); Pfam (Protein family); KOG/COG (Clusters of Orthologous Groups of proteins); Swiss-Prot (A manually annotated and reviewed protein sequence database); KO (KEGG Ortholog database); GO (Gene Ontology).

\section{Reads mapping to the reference genome}

Sequencing-received raw image data was transformed by base calling into sequence data. Prior to mapping reads to the reference database, we filtered all sequences to remove low quality sequences. A preprocessed database of nucleotide tag was created using our transcriptome reference database. The clean reads of the samples were mapped to the reference sequence of Uncaria transcriptome data, and obtained from the annotation information of each sample using RSEM software.

\section{Quantification of gene expression level}

HTSeq v0.5.3 was used to count the reads numbers mapped to each gene. And then RPKM of each gene was calculated based on the length of the gene and reads count mapped to this gene. RPKM, Reads Per Kilobase of exon model per Million mapped reads, considers the effect of sequencing depth and gene length for the reads count at the same time, and is currently the most commonly used method for estimating gene expression levels [47].

\section{Differential expression analysis}

Prior to differential gene expression analysis, for each sequenced library, the read counts were adjusted by edgeR program package through one scaling normalized factor. Differential expression analysis of two conditions was performed using the DEGseq R package (1.12.0). The $\mathrm{P}$ values were adjusted using the Benjamini \& Hochberg method [51]. Corrected P-value of 0.005 and $\log 2$ (Fold change) of 1 were set as the threshold for significantly differential expression.

\section{GO and KEGG enrichment analysis of differentially expressed genes}

Gene Ontology (GO) enrichment analysis of differentially expressed genes was implemented by the GOseq $\mathrm{R}$ package, in which gene length bias was corrected. GO terms with corrected P-value less than 0.05 were considered significantly enriched by differential expressed genes.

KEGG is a database resource for understanding highlevel functions and utilities of the biological system, such as the cell, the organism and the ecosystem, from molecular-level information, especially large-scale molecular datasets generated by genome sequencing and other high-throughput experimental technologies (http://www.genome.jp/kegg/). We used KOBAS software to test the statistical enrichment of differential expression genes in KEGG pathways. 


\section{Quantitative PCR}

RNA for quantitative PCR analysis was the same samples as used for DGE. Primers were designed using Primer 5.0, and shown in Additional file 1: Table S10. Reverse transcription used PrimerScoript RT reagent Kit With gDNA eraser (perfect Real Time) (TaKaRa, RR047A) and Random 6 mers primer obtained cDNA. The standard curve was constructed using cDNA generated 1:5 with $\mathrm{H}_{2} \mathrm{O}$. The cDNA was diluted in the $1: 20$ ratio as the sample reaction solution.

Quantitative PCR reactions used ABI7500 quantitative PCR, and the relative standard curve method was adopted to analyze the relative expression of genes. This study used SYBR $^{\circledR}$ Premix Ex TaqTM II (TaKaRa, DRR820A) kit, which reaction system was $25 \mu \mathrm{L}$, final concentration of primers was $0.2 \mu \mathrm{mol} \cdot \mathrm{L}^{-1}$, template was $1.0 \mu \mathrm{L}$, ROX Reference Dye II was $0.5 \mu \mathrm{L}, \mathrm{H}_{2} \mathrm{O}$ was used for complement system, and set up six repeats. Negative control used $1.0 \mu \mathrm{L}$ water instead of the template. Reaction conditions were $95^{\circ} \mathrm{C}$ for $5 \mathrm{~s}$ in order to activate enzyme reaction. Two step cycles were then used; $95^{\circ} \mathrm{C}$ for $5 \mathrm{~s}$, then $60^{\circ} \mathrm{C}$ for $30 \mathrm{~s}, 40$ cycles; solubility curve conditions were $95^{\circ} \mathrm{C}$ for $15 \mathrm{~s}, 60^{\circ} \mathrm{C}$ for $1 \mathrm{~min}$, $95^{\circ} \mathrm{C}$ for $30 \mathrm{~s}, 60^{\circ} \mathrm{C}$ for $15 \mathrm{~s}$.

\section{Availability of supporting data}

The data sets supporting the results of this article are available in the BioProject (BioProject:PRJNA222278, PRJNA222280, PRJNA222281, PRJNA222282) repository of the National Center for Biotechnology Information: http://www.ncbi.nlm.nih.gov/bioproject/?term=222278, http://www.ncbi.nlm.nih.gov/bioproject/?term=222280, http://www.ncbi.nlm.nih.gov/bioproject/?term=222281, http://www.ncbi.nlm.nih.gov/bioproject/?term=222282.

\section{Additional files}

Additional file 1: Table S1. Summary for the Uncaria rhynchophylla transcriptome. Table S2. The length statistics of the assembled transcripts and unigenes. Table S3. Coding genes and CDS involved in TIAs biosynthesis by $\mathrm{Nr}$ database annotation. Table S4. (A) Coding genes of cytochrome P450 protein. (B) Coding genes of methyltransferase. (C) Coding genes of isomerase. Table S5. Encoding genes involved in TIAs biosynthesis by KO database annotation. Table S6. RPKM and up/down-regulated expression of encoding genes involved in TIAs biosynthesis by $\mathrm{Nr}$ database annotation. Table S7. Up/down regulated expression of encoding genes involved in TIAs biosynthesis by KO database annotation. Table S8. (A) Differential expression analysis of cytochrome P450 genes. (B) Differential expression analysis of methyltransferase genes. (C) Differential expression analysis of isomerase genes. Table S9. Unigene and amino acid sequences of RIN and IRN biosynthetic encoding genes in the late step. Table S10. The quantitative PCR primers of candidate genes.

Additional file 2: Figure S1. Error rate distribution of Capsule transcriptome sequencing. The $x$-axis indicates the nucleotide position of reads. The $y$-axis indicates the single-base error rate. 1-100 bp is a first terminal sequencing error rate distribution for the two-terminal sequencing reads, $100-200 \mathrm{bp}$ is the other terminal sequencing error rate distribution. Figure S2. G/C content distribution. $0-100$ bp is the GC content distribution of the first reads sequencing in paired-end sequencing, 100-200 bp is the $\mathrm{GC}$ content distribution of the other reads sequencing.

Figure S3. (A) GO classification of differentially expressed genes in capsule 2 vs capsule 1. (B) GO classification of differentially expressed genes in capsule 3 vs capsule 1. (C) GO classification of differentially expressed genes in capsule 3 vs capsule 2. Figure S4. (A) KEGG pathway enrichment scatter diagram in capsule 2 vs capsule 1. (B) KEGG pathway enrichment scatter diagram in capsule 3 vs capsule 1. (C) KEGG pathway enrichment scatter diagram in capsule 3 vs capsule 2.

\section{Abbreviations}

RIN: Rhynchophylline; IRN: Isorhynchophylline; TIAs: Terpene indole alkaloids; Nr: Non-redundant protein sequences; KO: Kyoto Encyclopedia of Genes and Genomes Ortholog database; CYP450: Cytochrome P450; DGE: Digital gene expression profile; $A \beta$ : $\beta$-amyloid protein; MEP/DOXP: Pathway triose phosphate/pyruvate or "non-mevalonate" pathway; IPP: Isopentenyl diphosphate; G10H: Geraniol-10-hydroxylase; LAMT: Loganic acid methyltransferase; SLS: Secologanin synthase; STR: Strictosidine synthase; Swiss Prot: A manually annotated and reviewed protein sequence database; Nt: NCBI nucleotide sequences; Pfam: Protein family; COG: Clusters of Orthologous Groups of proteins; GO: Gene Ontology; ORF: Open Reading Frame; CDS: Coding Sequence; TDC: Tryptophan decarboxylase; Ref: Reference sequence; RPKM: Reads Per Kilo bases per Million mapped Reads.

\section{Competing interests}

The authors declare that they have no competing interests.

\section{Authors' contributions}

QQG conceived the study, designed and built the cDNA library, participated in data analysis, and drafted the manuscript. WZ and XJM initiated the project, helped to conceive the study, and participated in the design and coordination. SGW participated in experiment materials preparation. PW, DYQ, IWW, TQ, LJL and SKD participated in manuscript formatting and editing. All authors read and approved the final manuscript.

\section{Acknowledgements}

This work was supported by the central project of significant increase, decrease and support. (Grant No. 2060302) and Guangxi National Science Foundation of China (grant No. 2012GXNSFBA053118).

\section{Author details}

${ }^{1}$ College of Agriculture, Northeast Agricultural University, Harbin 150030, China. ${ }^{2}$ College of Life Science, Agriculture and Forest, Qiqihar University, Qiqihar 161006, China. ${ }^{3}$ Institute of Medicinal Plant Development, Chinese Academy of Medical Sciences, Beijing 100193, China. ${ }^{4}$ Yunnan Branch Institute of Medicinal Plant Development, Chinese Academy of Medical Sciences, Jinghong 530023, China. ${ }^{5}$ Guangxi Medicinal Botanical Garden, Nanning 530023, China. 'State Key Laboratory of Tree Genetics and Breeding, The Research Institute of Forestry, Chinese Academy of Forestry, Beijing 100091, China. ${ }^{7}$ CSIRO Plant Industry, PO Box 1600, Canberra ACT 2001, Australia. ${ }^{8}$ Hunan Agricultural University, Changsha 410128, China.

Received: 31 August 2013 Accepted: 4 August 2014 Published: 12 August 2014

\section{References}

1. Ferri CP, Prince M, Brayne C, Brodaty H, Fratiglioni L, Ganguli M, Hall K, Hasegawa K, Hendrie H, Huang YQ, Jorm A, Mather C, Menezes PR, Rimmer E, Scazufca M: Global prevalence of dementia: a Delphi consensus study. Lancet 2005, 366:2112-2117.

2. Lane RM, He Y: Butyrylcholinesterase genotype and gender influence Alzheimer's disease phenotype. Alzheimers Dement 2013, 9:e1-e73.

3. Terry AV Jr, Callahan PM, Hall B, Webster SJ: Alzheimer's disease and age-related memory decline (preclinical). Pharmacol Biochem Behav 2011, 99:190-210.

4. Ehrman TM, Barlow DJ, Hylands PJ: Phytochemical databases of Chinese herbal constituents and bioactive plant compounds with known target specificities. J Chem Inf Model 2007, 47:254-263. 
5. Fujiwara H, Iwasaki K, Furukawa K, Seki T, He M, Maruyama M, Tomita N, Kudo Y, Higuchi M, Saido TC, Maeda S, Takashima A, Hara M, Ohizumi Y, Arai H: Uncaria rhynchophylla, a chinese medicinal herb, has potent anti-aggregation effects on alzheimer's $\beta$-amyloid proteins. J Neurosci Res 2006, 84:427-433.

6. Xian YF, Lin ZX, Zhao M, Mao QQ, Ip SP, Che CT: Uncaria rhynchophylla ameliorates cognitive deficits induced by D-galactose in mice. Planta Med 2011, 77:1977-1983.

7. Yuan D, Ma B, Yang JY, Xie YY, Wang L, Zhang LJ, Kano Y, Wu CF: Antiinflammatory effects of rhynchophylline and isorhynchophylline in mouse N9 Microglial cells and the molecular mechanism. Int Immunopharmacol 2009, 9:1549-1554.

8. Xian YF, Lin ZX, Mao QQ, Hu Z, Zhao M, Che CT, Ip SP: Bioassay-guided isolation of neuroprotective compounds from Uncaria rhynchophylla against beta-amyloid-induced neurotoxicity. Evid-Based Compl Alt 2012, 2012:1-8

9. Xian YF, Lin ZX, Mao QQ, Ip SP, Su ZR, Lai XP: Protective effect of isorhynchophylline against $\beta$-amyloid-induced neurotoxicity in $P C_{12}$ cells. Cell Mol Neurobiol 2012, 3:353-360.

10. Stöckigta J, Barlebenb L, Panjikarc S, Lorisa EA: 3D-structure and function of strictosidine synthase-the key enzyme of monoterpenoid indole alkaloid biosynthesis. Plant Physiol Biochem 2008, 46:340-355.

11. Pasquali G, Porto DD, Fett-Neto AG: Metabolic engineering of cell cultures versus whole plant complexity in production of bioactive monoterpene indole alkaloids: recent progress related to old dilemma. J Biosci Bioeng 2006, 4:287-296.

12. Fawcett SL, Grant I, Hall PN, Kelsall AWR, Nicholson JC: Vincristine as a treatment for a large haemangioma threatening vital functions. Br J Plast Surg 2004, 57:168-171.

13. Casado P, Zuazua-Villar P, del Valle E, Martı'nez-Campa C, Lazo PS, Ramos S: Vincristine regulates the phosphorylation of the antiapoptotic protein HSP27 in breast cancer cells. Cancer Lett 2007, 247:273-282.

14. Tarlaci S: Vincristine-induced fatal neuropathy in non-Hodgkin's lymphoma. Neurotoxicology 2008, 29:748-749.

15. Chiu WH, Luo SJ, Chen CL, Cheng JH, Hsieh CY, Wang CY, Huang WC, Su WC, Lin CF: Vinca alkaloids cause aberrant ROS-mediated JNK activation, Mcl-1 downregulation, DNA damage, mitochondrial dysfunction, and apoptosis in lung adenocarcinoma cells. Biochem Pharmacol 2012, 9:1159-1171.

16. Rolf $\mathrm{S}$, Bruns HJ, Wichter T, Kirchhof P, Ribbing M, Wasmer K, Paul M, Breithardt G, Haverkamp W, Eckardt L: The ajmaline challenge in Brugada syndrome: diagnostic impact, safety, and recommended protocol. Eur Heart J 2003, 24:1104-1112.

17. Wolpert C, Echternach C, Veltmann C, Antzelevitch C, Thomas GP, Spehl S, Streitner F, Kuschyk J, Schimpf R, Haase KK, Borggrefe M: Intravenous drug challenge using flecainide and ajmaline in patients with Brugada syndrome. Heart Rhythm 2005, 3:254-260.

18. Swann AC, Birnbaum D, Jagar AA, Dougherty DM, Moeller FG: Acute yohimbine increases laboratory-measured impulsivity in normal subjects. Biol Psychiatry 2005, 57:1209-1211.

19. Farouk M, El-Aziz LA, El-Gindy AE, Shokny E: Validated methods for determination of yohimbine hydrochloride in the presence of its degradation products. Bull Fac Pharm Cairo Univ 2011, 49:67-79.

20. Subramanian N, Sundaraganesan N, Sudha S, Aroulmoji V, Sockalingam GD, Bergamin M: Experimental and theoretical investigation of the molecular and electronic structure of anticancer drug camptothecin. Spectrochim Acta A Mol Biomol Spectrosc 2011, 78:1058-1067.

21. Krishnakumar G, Rameshkumar KB, Srinivas P, Satheeshkumar K, Krishnan PN: Estimation of camptothecin and pharmacological evaluation of Ophiorrhiza prostrata D. Don and Ophiorrhiza mungos L. Asian Pac J Trop Biomed 2012, 2:S727-S731.

22. Kofoed PE, Có F, Johansson P, Dias F, Cabrai C, Hedegaard K, Aaby P, Rombo L: Treatment of uncomplicated malaria in children in GuineaBissau with chloroquine, quinine, and sulfadoxine-pyrimethamine. Trans R Soc Trop Med Hyg 2002, 3:304-309.

23. Eltahir HG, Omer AA, Mohamed AA, Adam I: Comparison of artesunate and quinine in the treatment of Sudanese children with severe Plasmodium falciparum malaria. Trans R Soc Trop Med Hyg 2010, 10:684-686.

24. Contin A, van der Heijden R, Lefeber AWM, Verpoorte R: The iridoid glucoside secologanin is derived from the novel triose phosphate/ pyruvate pathway in a Catharanthus roseus cell culture. FEBS Lett 1998 434:413-416.

25. Yamazaki Y, Kitajima M, Arita M, Takayama H, Sudo H, Yamazaki M, Aimi N, Saito K: Biosynthesis of camptothecin in silico and in vivo tracer study from $\left[1-{ }^{13} \mathrm{C}\right]$ glucose. Plant Physiol 2004, 134:161-170.

26. Collu G, Unver N, Peltenburg-Looman AMG, van der Heijden R, Verpoorte $R$, Memelink J: Geraniol 10-hydroxylase1, a cytochrome P450 enzyme involved in terpenoid indole alkaloid biosynthesis. FEBS Lett 2001, 508:215-220.

27. Collu G, Garcia A, van der Heijden R, Verpoorte R: Activity of the cytochrome P450 enzyme geraniol 10-hydroxylase and alkaloid production in plant cell cultures. Plant Sci 2002, 1:165-172.

28. Irmler S, Schröder G, St-Pierre B, Crouch NP, Hotze M, Schmidt J, Strack D, Matern U, Schröder J: Indole alkaloid biosynthesis in Catharanthus roseus: new enzyme activities and identification of cytochrome P450 CYP72A1 as secologanin synthase. Plant J 2000, 6:797-804.

29. Yamamoto $\mathrm{H}$, Katano N, Ooi A, Inoue K: Secologanin synthase which catalyzes the oxidative cleavage of loganin into secologanin is a cytochrome P450. Phytochemistry 2000, 53:7-12

30. Geu-Flores F, Sherden NH, Courdavault V, Burlat V, Glenn WS, Wu C, Nims E, Cui Y, O'Connor SE: An alternative route to cyclic terpenes by reductive cyclization in iridoid biosynthesis. Nature 2012, 492:138-142.

31. Asada K, Salim V, Masada-Atsumi S, Edmunds E, Nagatoshi M, Terasaka K, Mizukami H, De Luca V: A 7-deoxyloganetic acid glucosyltransferase contributes a key step in Secologanin Biosynthesis in Madagascar periwinkle. Plant Cell 2013, 25:4123-4134.

32. Salim V, Yu F, Altarejos-Caballero J, De Luca V: Virus induced gene silencing identifies Catharanthus roseus 7-deoxyloganic acid 7-hydroxylase, a step in iridoid and monoterpenoid indole alkaloid biosynthesis. Plant J 2013 http://dx.doi.org/10.1111/tpj.12330.

33. Guirimand G, Guihur A, Ginis O, Poutrain P, Héricourt F, Oudin A, Lanoue A St-Pierre B, Burlat V, Courdavault V: The subcellular organization of strictosidine biosynthesis in Catharanthus roseus epidermis highlights several trans-tonoplast translocations of intermediate metabolites. FEBS J 2011, 278:749-763.

34. Canel C, Lopes-Cardoso MI, Whitmer S, van der Fits L, Pasquali G, van der Heijden R, Hoge JHC, Verpoorte R: Effects of over-expression of strictosidine synthase and tryptophan decarboxylase on alkaloid production by cell cultures of Catharanthus roseus. Planta 1998, 205:414-419.

35. Oudin A, Courtois M, Rideau M, Clastre M: The iridoid pathway in Catharanthus roseus alkaloid biosynthesis. Phytochem Rev 2007, 6:259-276.

36. Salim V, De Luca V: Towards complete elucidation of monoterpene indole alkaloid biosynthesis pathway: Catharanthus roseus as a pioneer system. Adv Bot Res 2013, 68:1-37.

37. Salim V, Wiens B, Masada-Atsumi S, Yu F, De Luca V: 7-Deoxyloganetic acid synthase catalyzes a key 3 step oxidation to form 7-deoxyloganetic acid in Catharanthus roseus iridoid biosynthesis. Phytochemistry 2014, 101:23-31.

38. Erlich Y, Mitra PP, dela Bastide M, McCombie WR, Hannon GJ: Alta-Cyclic: a self-optimizing base caller for next-generation sequencing. Nat Methods 2008, 8:679-682

39. Jiang $L$, Schlesinger F, Davis CA, Zhang Y, Li R, Salit M, Gingeras TR, Oliver B: Synthetic spike-in standards for RNA-seq experiments. Genome Res 2011, 21:1543-1551.

40. Hansen KD, Brenner SE, Dudoit S: Biases in Illumina transcriptome sequencing caused by random hexamer priming. Nucleic Acids Res 2010, 38:e131-e131.

41. Grabherr MG, Haas BJ, Yassour M, Levin JZ, Thompson DA, Amit I, Adiconis X, Fan L, Raychowdhury R, Zeng Q, Chen ZH, Mauceli E, Hacohen N, Gnirke A, Rhind N, di Palma F, Birren BW, Nusbaum C, Lindblad-Toh K, Friedman N, Regev A: Full-length transcriptome assembly from RNA-Seq data without a reference genome. Nat Biotechnol 2011, 29:644-652.

42. Kumar S, Blaxter ML: Comparing de novo assemblers for 454 transcriptome data. BMC Genomics 2010, 11:571.

43. Götz S, García-Gómez JM, Terol J, Williams TD, Nagaraj SH, Nueda SH, Robles M, Talón M, Dopazo J, Conesa A: High-throughput functional annotation and data mining with the Blast2GO suite. Nucleic Acids Res 2008, 36:3420-3435.

44. Iseli C, Jongeneel CV, Bucher P: ESTScan: a program for detecting, evaluating, and reconstructing potential coding regions in EST sequences. Proc Int Conf Intell Syst Mol Biol 1999, 138-148. 
45. O' Connor SE, Maresh JJ: Chemistry and biology of monoterpene indole alkaloid biosynthesis. Nat Prod Rep 2006, 23:532-547.

46. Li B, Dewey CN: RSEM: accurate transcript quantification from RNA-Seq data with or without a reference genome. BMC Bioinformatics 2011, 12:323.

47. Mortazavi A, Williams BA, McCue K, Schaeffer L, Wold B: Mapping and quantifying mammalian transcriptomes by RNA-Seq. Nat Methods 2008, 5:621-628.

48. Robinson MD, Oshlack A: A scaling normalization method for differential expression analysis of RNA-seq data. Genome Biol 2010, 11:R25.

49. Anders $S$, Huber W: Differential expression analysis for sequence count data. Genome Biol 2010, 11:R106.

50. Storey JD, Tibshirani R: Statistical significance for genomewide studies. Proc Natl Acad Sci U S A 2003, 16:9440-9445.

51. Benjamini $Y$, Hochberg $Y$ : Controlling the false discovery rate: a practical and powerful approach to multiple testing. J R Statist Soc B 1995, 1:289-300.

52. Young MD, Wakefield MJ, Smyth GK, Oshlack A: Gene ontology analysis for RNA-seq: accounting for selection bias. Genome Biol 2010, 11:R14.

53. Tang Q, Ma XJ, Mo CM, Wilson IW, Song C, Zhao H, Yang YF, Fu W, Qiu DY: An efficient approach to finding Siraitia grosvenorii triterpene biosynthetic genes by RNA-seq and digital gene expression analysis. BMC Genomics 2011, 12:343.

54. Coon MJ: Cytochrome P450: nature's most versatile biological catalyst. Annu Rev Pharmacol Toxicol 2005, 45:1-25.

55. Morant M, Bak S, Moller BL, Werck-Reichhart D: Plant cytochromes P450: tools for pharmacology, plant protection and phytoremediation. Curr Opin Biotechnol 2003, 14:151-162.

56. Guttikonda SK, Trupti J, Bisht NC, Chen H, An YQC, Pandey S, Xu D, Yu O: Whole genome co-expression analysis of soybean cytochrome P450 genes identifies nodulationspecific P450 monooxygenases. BMC Plant Biol 2010, 10:243.

57. Devi BS, Kim YJ, Sathiyamoorthy S, Khorolragchaa A, Gayathri S, Parvin S, Yang DU, Selvi SK, Lee OR, Lee S, Yang DC: Classification and characterization of putative cytochrome $\mathrm{P} 450$ genes from panax ginseng C. A. Meyer. Biochemistry (Mosc) 2011, 12:1347-1359.

58. Li DX, Du XJ, Zhao XF, Wang JX: Cloning and expression analysis of an O-methyltransferase (OMT) gene from Chinese shrimp, Fenneropenaeus chinensis. Fish Shellfish Immunol 2006, 21:284-292.

59. Kota P, Guo DJ, Zubiet C, Noel J, Dixon RA: O-Methylation of benzaldehyde derivatives by "lignin specific" caffeic acid 3-O-methyltransferase. Phytochemistry 2004, 65:837-846.

60. Li Y, Luo HM, Sun C, Song JY, Sun YZ, Wu Q, Wang N, Yao H, Steinmetz A, Chen SL: EST analysis reveals putative genes involved in glycyrrhizin biosynthesis. BMC Genomics 2010, 11:268.

61. Logacheva MD, Kasianov AS, Vinogradov DV, Samigullin TH, Gelfand MS, Makeev VJ, Penin AA: De novo sequencing and characterization of floral transcriptome in two species of buckwheat (Fagopyrum). BMC Genomics 2011, 12:30.

62. Al-Dous EK, George B, Al-Mahmoud ME, Al-Jaber MY, Wang H, Salameh YM, Al-Azwani EK, Chaluvadi S, Pontaroli AC, DeBarry J, Arondel V, Ohlrogge J, Saie IJ, Suliman-Elmeer KM, Bennetzen JL, Kruegger RR, Malek JA: De novo genome sequencing and comparative genomics of date palm (Phoenix dactylifera). Nat Biotechnol 2011, 6:521-527.

63. Gahlan $P$, Singh HR, Shankar R, Sharma N, Kumari A, Chawla V, Ahuja PS, Kumar S: De novo sequencing and characterization of Picrorhiza kurrooa transcriptome at two temperatures showed major transcriptome adjustments. BMC Genomics 2012, 13:126.

64. Ashrafi H, Hill T, Stoffel K, Kozik A, Yao JQ, Chin-Wo SR, Deynze AV: De novo assembly of the pepper transcriptome (Capsicum annuum): a benchmark for in silico discovery of SNPs, SSRs and candidate genes. BMC Genomics 2012, 13:571.

65. Imelfort M, Edwards D: De novo sequencing of plant genomes using second-generation technologies. Brief Bioinform 2009, 6:609-618.

66. Wang Z, Gerstein M, Snyder M: RNA-Seq: a revolutionary tool for transcriptomics. Nat Rev Genet 2009, 10:57-63.

67. Sun C, Li Y, Wu Q, Luo HM, Sun YZ, Song JY, Lui EMK, Chen SL: RDe novo sequencing and analysis of the American ginseng root transcriptome using a GS FLX Titanium platform to discover putative genes involved in ginsenoside biosynthesis. BMC Genomics 2010, 11:262
68. Annadurai RS, Jayakumar V, Mugasimangalam RC, Katt MA, Anand S, Gopinathan S, Sarma SP, Fernandes SJ, Mullapudi N, Murugesan S, Rao SN: Next generation sequencing and de novo transcriptome analysis of Costus pictus D. Don, a non-model plant with potent anti-diabetic properties. BMC Genomics 2012, 13:663.

69. Zhou YJ, Gao F, Liu R, Feng JC, Li HJ: De novo sequencing and analysis of root transcriptome using 454 pyrosequencing to discover putative genes associated with drought tolerance in Ammopiptanthus mongolicus. BMC Genomics 2012, 13:266.

70. Eveland AL, Satoh-Nagasawa N, Goldshmidt A, Meyer S, Beatty M, Sakai H, Ware D, Jackson D: Digital gene expression signatures for maize development. Plant Physiol 2010, 11(154):1024-1039.

71. Verpoorte R, Contin A, Memelink J: Biotechnology for the production of plant secondary metabolites. Phytochem Rev 2002, 1:13-25.

72. van Der Heijden R, Jacobs DI, Snoeijer W, Hallard D, Verpoorte R: The Catharanthus Alkaloids:Pharmacognosy and Biotechnology. Curr Med Chem 2004, 5:607-628.

73. Glenn WS, Runguphan W, O' Connor SE: Recent progress in the metabolic engineering of alkaloids in plant systems. Curr Opin Biotechnol 2013, 24:354-365.

74. Tiwari R, Awasthi A, Mall M, Shukla AK, Satya Srinivas KVN, Syamasundar KV, Kalra A: Bacterial endophyte-mediated enhancement of in planta content of key terpenoid indole alkaloids and growth parameters of Catharanthus roseus. Ind Crop Prod 2013, 43:306-310.

75. Suttipanta N, Pattanaik S, Gunjan S, Xie CH, Littleton J, Yuan L: Promoter analysis of the Catharanthus roseus geraniol 10-hydroxylase gene involved in terpenoid indole alkaloid biosynthesis. Biochim Biophys Acta 2007, 1769:139-148.

76. Memelink J: The use of genetics to dissect plant secondary pathways. Curr Opin Plant Biol 2005, 8:230-235.

77. Montiel G, Breton C, Thiersault M, Burlat V, Jay-Allemand C, Gantet P. Transcription factor Agamous-like 12 from arabidopsis promotes tissuelike organization and alkaloid biosynthesis in Catharanthus roseus suspension cells. Metab Eng 2007, 9:125-132.

78. Chemler JA, Koffas MA: Metabolic engineering for plant natural product biosynthesis in microbes. Curr Opin Biotechnol 2008, 19:597-605.

79. Cordell GA: Fifty years of alkaloid biosynthesis in Phytochemistry. Phytochemistry 2013, 91:29-51.

80. Guo Q, Ma X, Jin'e F, Wei Z: Study on accumulation feature of rhynchophylline and isorhynchophylline in Uncaria rhynchophylla. Res J Biotechnol 2014, 9:1-6.

81. Guo QQ, Ma XJ, Wei SG, Bai LH, Fu JE, Dong SK, Liu LJ, Zu W: Isolation of RNA from uncaria with medicinal plant. Crops 2013, 5:80-83.

doi:10.1186/1471-2164-15-676

Cite this article as: Guo et al:: De novo transcriptome sequencing and digital gene expression analysis predict biosynthetic pathway of rhynchophylline and isorhynchophylline from Uncaria rhynchophylla, a non-model plant with potent anti-alzheimer's properties. BMC Genomics 2014 15:676.

\section{Submit your next manuscript to BioMed Central and take full advantage of:}

- Convenient online submission

- Thorough peer review

- No space constraints or color figure charges

- Immediate publication on acceptance

- Inclusion in PubMed, CAS, Scopus and Google Scholar

- Research which is freely available for redistribution 